\title{
Paraneoplastischer Pemphigus bei hochmalignem Non-Hodgkin-Lymphom bei einem Patienten mit Psoriasis pustulosa unter Therapie mit Infliximab
}

\author{
Paraneoplastic Pemphigus Due to High Malignant non Hodgkin Lymphoma in a Patient with Pustular Psoriasis \\ Treated with Infliximab
}

Autoren

Institute
A. Schöberl' ${ }^{1}$, C. Allmacher ${ }^{1}$, N. Flegl', B. Krahl'², D. Krahl'², U. Amon ${ }^{1}$

PsoriSol Hautklinik, Hersbruck (Metropolregion Nürnberg)

2 Institut für Dermatohistologie, Heidelberg
Bibliografie

DOI http://dx.doi.org/ $10.1055 / \mathrm{s}-0029-1215213$

Online-Publikation: 8. 10. 2009

Akt Dermatol 2010; 36 :

129-132 @ Georg Thieme

Verlag KG Stuttgart · New York ISSN 0340-2541

Korrespondenzadresse

Prof. Dr. med. habil.

Ulrich Amon

PsoriSol Hautklinik GmbH Fachklinik für Dermatologie und Allergologie

Mühlstraße 31

91217 Hersbruck

amon@psorisol.de

\section{Zusammenfassung \\ $\nabla$}

Wir berichten über einen 54-jährigen Patienten mit Psoriasis pustulosa Typ Zumbusch, der erstmalig unter interner Therapie mit Infliximab an

\section{Anamnese}

$\nabla$

Der 54-jährige Patient, von Beruf Bauleiter, verheiratet, 3 Kinder, mit seit der Kindheit bekannter Psoriasis vulgaris mit chronisch-rezidivierendem Verlauf ohne Gelenkbeteiligung, wurde im Januar 2008 aufgrund einer postinfektiös infolge einer eitrigen Tracheobronchitis erstmalig aufgetretenen Psoriasis pustulosa Typ Zumbusch stationär mit deutlicher Leistungsminderung und einer ungewollten Gewichtsabnahme von $11 \mathrm{~kg}$ in $3 \mathrm{Wo}$ chen aufgenommen. Eine regelmäßige häusliche Medikation wurde nicht eingenommen.

\section{Diagnose, Therapie und Verlauf \\ $\nabla$}

Zum Zeitpunkt der stationären Aufnahme sahen wir einen wachen, bewusstseinsklaren Patienten in reduziertem Allgemeinzustand und mit normalem Ernährungszustand $(88 \mathrm{~kg}, 186 \mathrm{~cm}$ ). Über das gesamte Integument verteilt zeigten sich kokardenförmig angeordnete, teils mit hämorrhagischen Krusten belegte, erythematöse Plaques und multiple, bis stecknadelkopfgroße, teils konfluierende Pusteln. Axillär beidseits fanden sich flächige Erosionen mit schmierig-gelblichen Belägen, im Bereich der Mundschleimhaut fielen weiße, abwischbare Beläge auf.

Laborchemisch fand sich eine Leukozytose von $16000 / \mu l$ (ohne eine prästationär vorausgegangene systemische Steroidmedikation), im Differenzial-Blutbild betrug der Anteil der Neutrophilen $91 \%$ und der Lymphozyten $4 \%$. Serologisch be- einem paraneoplastischen Pemphigus vulgaris bei erstdiagnostiziertem Non-Hodgkin-Lymphom erkrankte, mit letztlich letalem Ausgang bei insgesamt progredientem Tumorleiden.

stand eine Hyperurikämie $(8,9 \mathrm{mg} / \mathrm{dl})$. Die restlichen Laborparameter (Blutbild, Differenzial-Blutbild, Kreatinin, GOT, GPT, rGT, Blutfette, Blutzucker, Bakterientiter) waren unauffällig. Laborchemisch ergab sich kein Hinweis auf eine HIV- oder Lues-Infektion.

Aufgrund des am Aufnahmetag klinisch eindeutigen Befundes einer generalisierten Psoriasis pustulosa ( $\triangle$ Abb. 1) und der Suberythrodermie leiteten wir eine interne Therapie mit Ciclosporin A $2,8 \mathrm{mg} / \mathrm{kg} \mathrm{KG}$ ein.

Extern erhielt der Patient Kaliumpermanganatumschläge und -vollbäder, intern eine orale Antibiose mit Erythromycin. In den folgenden Tagen des stationären Aufenthaltes zeigten sich im Verlauf zunehmend Pusteln am Oberkörper und an den Oberschenkeln. Bei insgesamt progredienten Psoriasiseffloreszenzen wurde schließlich die Ciclosporin A-Therapie auf $3,4 \mathrm{mg} / \mathrm{kg} \mathrm{KG}$ täglich erhöht.

Aufgrund einer jedoch im Verlauf zunehmenden Verschlechterung des Allgemeinzustandes wurde der Patient auf die internistische Intensivstation verlegt. Angesichts des ausgeprägten Hautbefundes erhielt der Patient schließlich nach interdisziplinärer, internistischer Rücksprache einmalig eine Infusion mit dem TNF- $\alpha$-Blocker Infliximab, wohingegen die Ciclosporin A-Dosis dann auf 2,3 mg/kg KG täglich reduziert wurde. Eine Antibiose mit Ciprofloxacin wurde eingeleitet, welche dann im Verlauf aufgrund des Auftretens septischer Organmanifestationen und Nachweis von MRSA um Vancomyin erweitert wurde. 


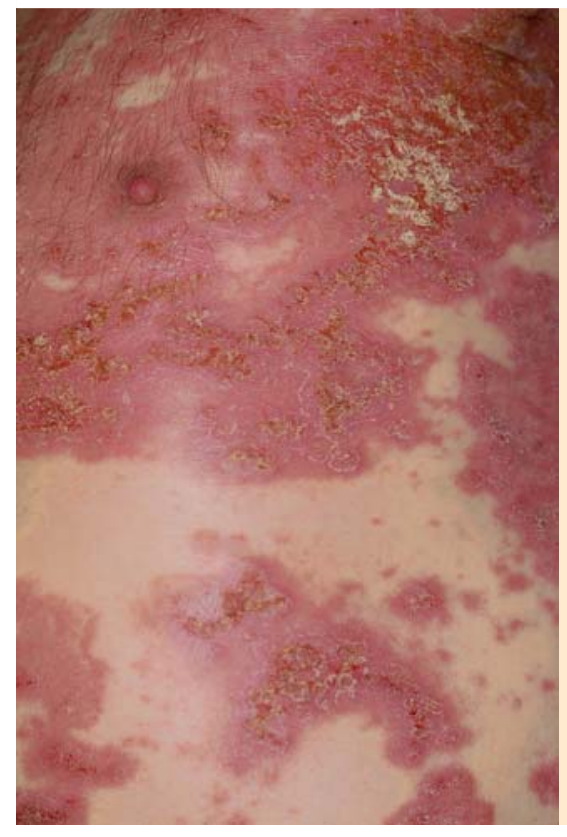

Abb. 1 Generalisierte Psoriasis pustulosa am Aufnahmetag.

Fünf Tage nach Verabreichen des Biologicals fanden sich im Bereich der Brust sowie der Augenlider erstmalig massive krustige Erosionen ( $\mathbf{A b b} . \mathbf{2}$ und $\mathbf{3}$ ).

Am Rücken und an den Oberschenkeln entwickelten sich erstmals multiple Erosionen mit teilweise noch erkennbarer dünner Blasendecke. In der indirekten Immunfluoreszenz auf Rattenharnblase fielen erhöhte Autoantikörper gegen Desmosomen (Titer 1:320) sowie eine schwach positive Reaktion gegen BP180 auf, der ELISA mit rekombinantem Desmoglein 1 bzw. 3 war in beiden Fällen positiv (138 U/ml bzw. $245 \mathrm{U} / \mathrm{ml}$ ). Die Histologie einer im Bereich des Dekolletés entnommenen Probebiopsie war vereinbar mit einem Pemphigus paraneoplasticus mit Lymphangiosis neoplastica ( $\bullet$ Abb. 4 und 5).

Unter partiell nekrotischer Epidermis mit konfluierenden Dyskeratosen und fokaler Akantholyse fand sich ein lichenoides gemischtzelliges entzündliches Infiltrat mit neutrophilen und weniger eosinophilen Granulozyten. Innerhalb vaskulärer Lumina ließ sich benachbart eine Population maligner Tumorzellen entsprechend einer Lymphangiosis neoplastica nachweisen. Die direkte Immunfluoreszenz mit Antikörpern gegen IgA, IgM, IgG, C3 und Fibrinogen zeigte bei massiven entzündlichen Sekundärveränderungen keine junktionalen oder epidermal-interzellulären Ablagerungen bzw. Markierung des Papillarkörpers. Unter Berücksichtigung der serologischen sowie histologischen und immunhistologischen Befunde konnte somit ein Pemphigus paraneoplasticus mit Lymphangiosis neoplastica diagnostiziert werden ${ }^{1}$.

Entsprechend erhielt der Patient nun hochdosiert Prednisolon intravenös, initial 2,8 mg/kg KG neben einer weiteren Reduktion der Ciclosporin A-Dosis.

Die parallel durchgeführte Tumorsuche inklusive histologischer Sicherung ergab ein follikuläres Lymphom Stadium III (zentroblastisch zentrozytisches Lymphom) mit in der Computertomografie pathologischen Lymphknotenpaketen mediastinal, links supraklavikulär, mesenterial, paraaortal und parakaval sowie pa-

\footnotetext{
${ }^{1}$ Wir danken herzlich für die unterstützende dermatohistologische Beurteilung des Labors Dr. Laaff, Freiburg.
}

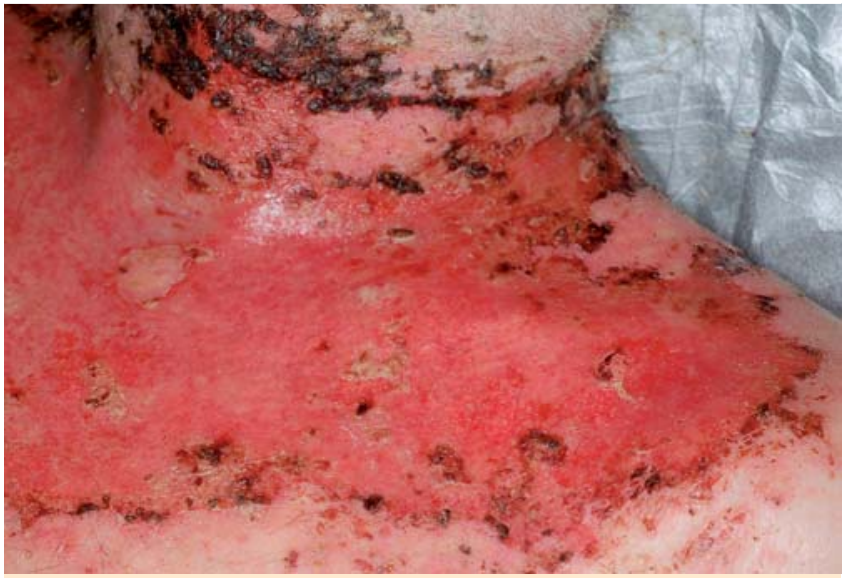

Abb. 2 Paraneoplastischer Pemphigus am Dekolleté.

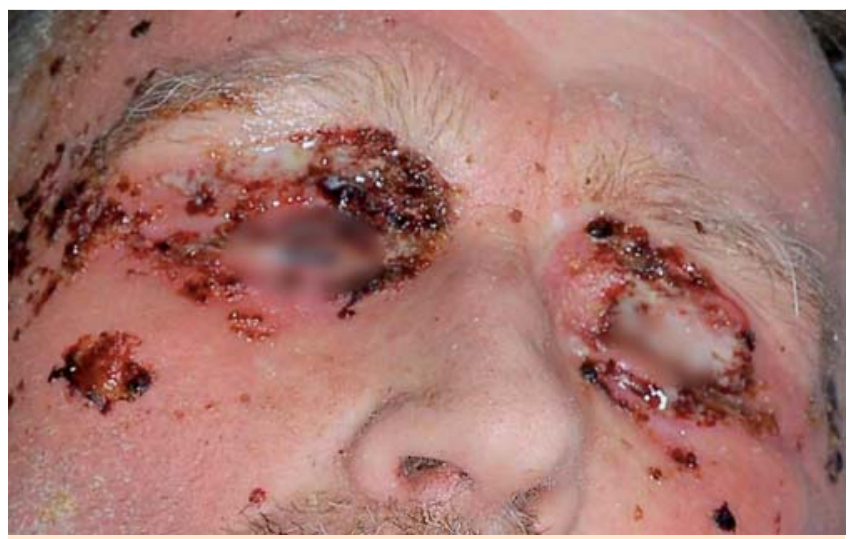

Abb. 3 Paraneoplastischer Pemphigus periorbikulär.

railiakal und retrokrural, sonografisch retroperitoneal im Ganzen vermessen ca. 11,4 $\times 2,9 \times 9,3 \mathrm{~cm}$.

Die im Februar 2008 konsekutiv begonnene Polychemotherapie nach dem R-CHOP-Schema führte zur deutlichen Besserung des Hautbefundes. Sonografische Verlaufskontrollen zeigten zunächst auch ein gutes Ansprechen der abdominellen Raumforderungen.

Der Patient erlitt allerdings während der Chemotherapie einen ischämischen Mediainsult links mit schlaffer Halbseitenlähmung rechtsseitig und motorischer Aphasie, sodass eine weitere Behandlung in einem neurologischen Zentrum unter interdisziplinärer neurologisch-internistisch-dermatologischer Zusammenarbeit erfolgte.

Im März 2008 wurde der Patient daher zunächst zur neurologischen Rehabilitationsbehandlung entlassen, eine Wiederholung der Chemotherapie nach dem R-CHOP-Schema alle drei Wochen erfolgte im Anschluss. Unter dieser war jedoch nun eine deutliche Tumorprogredienz zu verzeichnen. Der Patient verstarb im Mai 2008 an den Folgen des Tumorleidens.

\section{Diskussion \\ $\nabla$}

Der geschilderte Krankheitsverlauf ist komplex. Die ursprüngliche generalisierte Psoriasis pustulosa zeigte eine Progredienz unter Therapie mit Ciclosporin A, weshalb eine Behandlung mit Infliximab begonnen wurde. Der paraneoplastische Pemphigus mit wahrscheinlich kausalem Zusammenhang zu dem nachgewiese- 


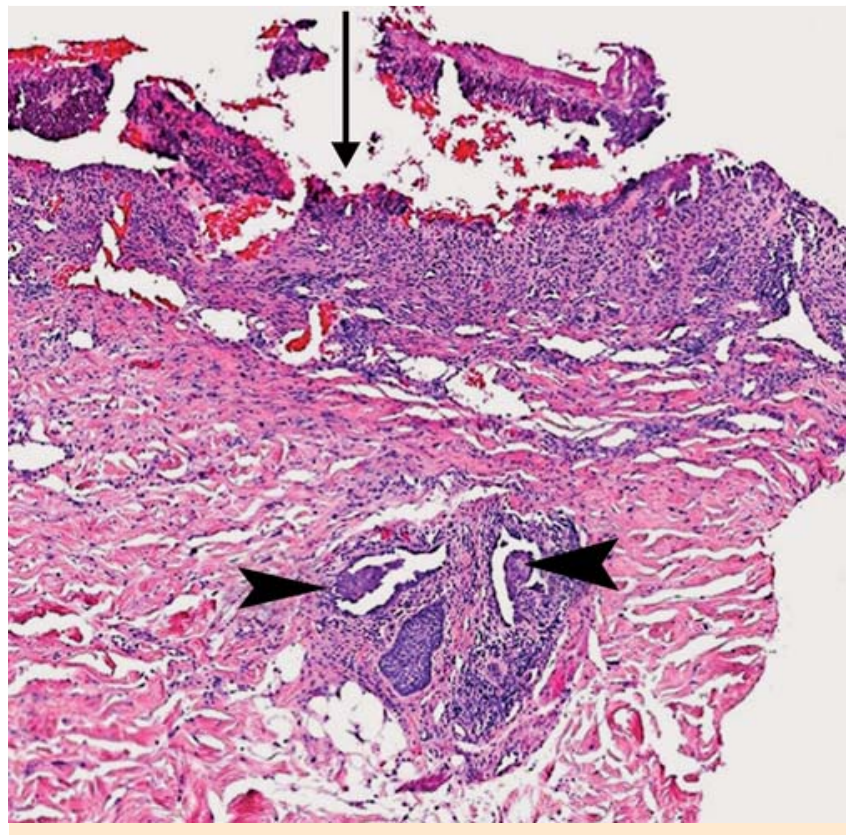

Abb. 4 Histologische Übersicht mit konfluenter Epidermisnekrose (Pfeil) und im mittleren Corium perifollikulär zwei dilatierten Gefäßen mit neoplastischer Zellpopulation des systemischen Lymphoms (Pfeilköpfe), $\mathrm{HE} \times 100$.

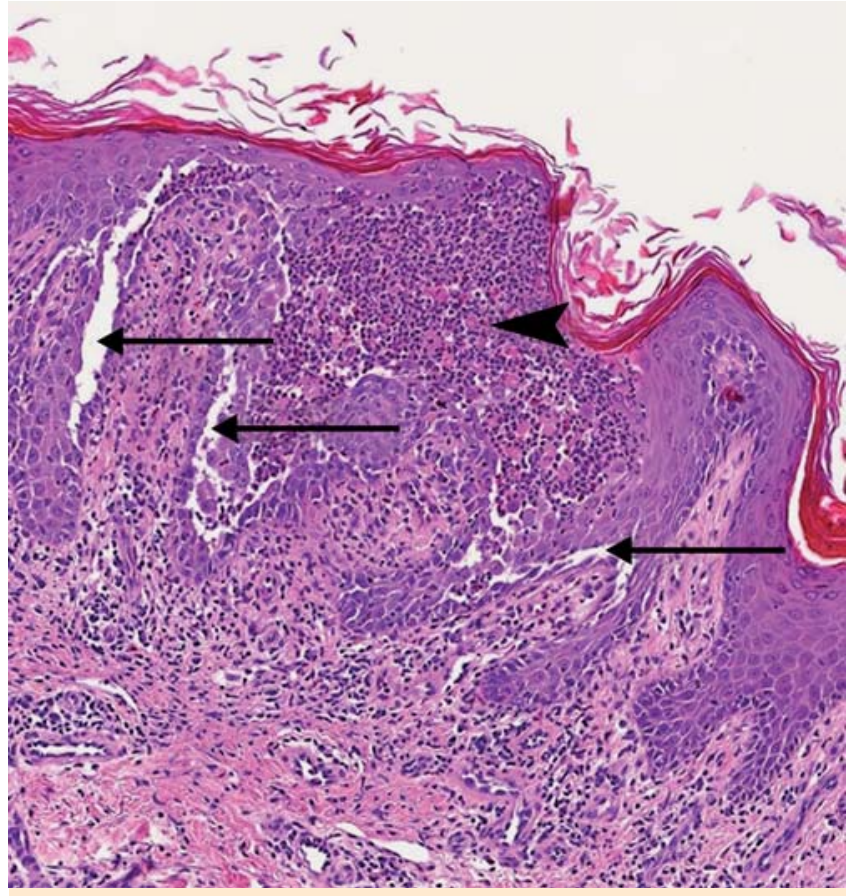

Abb. 5 Epidermis mit akantholytischer Blase (Pfeile) und Massen entzündlich überlagerter Dyskeratosen (Pfeilkopf), HE × 200.

nen hochmalignen Lymphom wirft auch Fragen zur möglichen Beeinflussung des Krankheitsverlaufs durch die Medikation auf. Allgemein gelten vorwiegend Non-Hodgkin-Lymphome, Thymome, chronisch lymphatische Leukämie und Morbus Castleman als mit einem paraneoplastischen Pemphigus assoziiert [1].

Es sind insgesamt mehrere Kasuistiken eines paraneoplastischen Pemphigus in Zusammenhang mit einem Lymphom beschrieben. Batista et al. beschrieben hierbei ebenso eine Assoziation eines paraneoplastischen Pemphigus mit einem B-Zell-Non-Hodgkin-
Lymphom bei einem 55-jährigen Patienten mit letztlich letalem Ausgang 2 Monate nach Diagnosestellung nach eingeleiteter Chemotherapie [2]. Auch Hoque et al. [3] und Duparc et al. [4] sahen einen Zusammenhang zwischen einem Non-Hodgkin-Lymphom und einem paraneoplastischen Pemphigus.

Bei Infliximab handelt es sich um einen monoklonalen chimären IgG1/kappa-AK gegen TNF- $\alpha$ mit gesicherter Wirkung bei Psoriasis vulgaris $[5,6]$. Auch zur Therapie der pustulösen Psoriasis wurde Infliximab inzwischen erfolgreich eingesetzt [7-9].

Für die Gruppe der TNF- $\alpha$-Blocker ergeben sich in der Literatur Hinweise auf ein erhöhtes Lymphomrisiko, obgleich widersprüchliche Daten vorliegen.

Wolfe und Michaud untersuchten in einer breiten US-Studie 13001 mit Biologicals behandelte Patienten mit rheumatoider Arthritis über 49000 Patientenjahre in den Jahren 1998-2005 hinsichtlich des Tumorrisikos [10]. Die erfassten Krebsraten wurden mit den Daten des US National Cancer Institute SEER (Surveillance, Epidemiology and End-Results) verglichen und zeigten letztlich eine Assoziation der Therapie mit Biologicals mit einem erhöhten Risiko für Hautkrebs, jedoch nicht für solide Tumoren oder lymphoproliferative Neoplasien. Auch Askling et al. konnten im Rahmen einer schwedischen Analyse mit 6604 mit TNF- $\alpha-A n-$ tagonisten therapierten Patienten mit rheumatoider Arthritis keine Assoziation mit einer weiteren Steigerung des bei Patienten mit rheumatoider Arthritis ohnehin bereits erhöhten Lymphomrisikos feststellen [11].

Gelfand et al. beschrieben ein ohnehin 2,95-faches relatives Lymphomrisiko für Psoriasispatienten allgemein im Vergleich zur Normalbevölkerung [12].

Brown u. Mitarb. registrierten im Rahmen der Überwachung von Gegenanzeigen von Etanercept und Infliximab zwischen Mai 1999 und Dezember 200026 Fälle lymphoproliferativer Erkrankungen, welche nach der Behandlung mit Etanercept in 18 Fällen und mit Infliximab in 8 Fällen auftraten [13]. Bei 81\% davon handelte es sich um Non-Hodgkin-Lymphome wie auch in unserer Kasuistik. Der zeitliche Abstand zwischen Beginn einer Therapie und dem Auftreten des Lymphoms wurde als kurzzeitig mit einem Median von 8 Wochen angegeben. In zwei Fällen, einer unter Etanercept, der andere unter Infliximab, wurde nach Therapieabbruch ein Lymphomregress beobachtet ohne Einsatz einer spezifischen zytotoxischen Therapie. Brown et al. bemerkten außerdem, dass die kurze Latenzperiode zwischen Therapiebeginn und Auftreten des Lymphoms in ähnlicher Weise bei immunsupprimierten Patienten nach Organtransplantation beobachtet wurde [14].

Somit stellt sich die Frage, inwiefern in unserem Fallbericht ein Zusammenhang zwischen Anti-TNF- $\alpha$-Therapie mit Infliximab und Lymphomentstehung oder-progress zu sehen ist. Schließlich ist auch Ciclosporin A selbst, das unser Patient zusätzlich erhalten hatte, bekanntlich mit einem erhöhten Lymphomrisiko assoziiert. Jedoch scheinen Lymphome offenbar nur bei langfristiger Ciclosporin-Exposition mit höheren Dosierungen, wie sie in der Transplantationsmedizin üblich sind, gehäuft aufzutreten [15].

Gemäß einer aktuellen retrospektiven Studie von 2008 mit 272 dermatologischen Patienten (davon 63 mit Psoriasis) scheint bei relativ kurzer Behandlungszeit (durchschnittliche Behandlungszeit mit Ciclosporin A von 8 Monaten) kein signifikant erhöhtes Lymphomrisiko zu bestehen [16]. Hierfür hatten Väkevä et al. die Patienten im Mittel 11 Jahre nachbeobachtet.

Diskussionswürdig ist für unsere Kasuistik in diesem Zusammenhang die Frage nach einem möglichen Zusammenhang zwischen der Kombinationstherapie von Infliximab und Ciclosporin 
und Lymphomentstehung oder -progress. Insgesamt sind zur Klärung derartiger Hypothesen weitere Fallbeobachtungen und Langzeitstudien abzuwarten.

\section{Fazit}

Der Fall unseres 54-jährigen Patienten, der unter sequentieller Therapie mit Ciclosporin A und Einmalgabe von Infliximab bei Psoriasis pustulosa Typ Zumbusch neben septischen Komplikationen einen paraneoplastischen Pemphigus vulgaris entwickelte, bei insgesamt progredient verlaufendem Tumorleiden mit Nachweis eines zentroblastisch-zentrozytischen Lymphoms im Stadium III, wirft erneut die Frage nach kausalem Zusammenhang zwischen Immunsuppression, Immunmodulation und Tumorprogress auf.

Angesichts der beschriebenen Zusammenhänge zwischen der Therapie mit Infliximab und einem erhöhten Infektionsrisiko, dem Auftreten von Lymphomen und der Induktion von Autoimmunerkrankungen erscheint in dieser Kasuistik eine Assoziation zwischen der Therapie und den angeführten Komplikationen zumindest nicht auszuschließen.

Angesichts der beschriebenen Risiken sollte in jedem Fall vor Einleitung einer Therapie eine sorgfältige Nutzen-Risiko-Abwägung erfolgen und das individuelle Risikoprofil des Patienten berücksichtigt werden.

\section{Abstract}

\section{Paraneoplastic Pemphigus Due to High Malignant non Hodgkin Lymphoma in a Patient with Pustular Psoriasis Treated with Infliximab \\ $\nabla$}

We present a 54-year old man with generalized pustular psoriasis who developed a paraneoplastic pemphigus due to a non Hodgkin lymphoma for the first time while being treated with infliximab. The progress of the lymphoma was lethal.

\section{Literatur}

1 Zhu X, Zhang B. Paraneoplastic Pemphigus. J Dermatol. 2007; 34: 503 511

2 Batista MD, Takano D, Lopes RD et al. Paraneoplastic pemphigus associated with non-Hodgkin lymphoma. Dermatol Online J 2008; 14: 11

3 Hoque SR, Black MM, Cliff S. Paraneoplastic pemphigus associated with CD20-positive follicular non-Hodgkin's lymphoma treated with rituximab: a third case resistant to rituximab therapy. Clin Exp Dermatol 2007; 32: $172-175$

4 Duparc A, Boivin S, Gilbert D et al. Paraneoplastic pemphigus with pemphigus vegetans-like lesions revealing non-hodgkin lymphoma. Eur J Dermatol 2006; 16: 698-699

5 Chaudhari U, Romano P, Mulcahy LD et al. Efficacy and safety of infliximab monotherapy for plaque-type psoriasis: a randomised trial. Lancet 2001; 357: $1842-1847$

6 Reich $K$, Nestle FO, Papp K et al. Infliximab induction and maintenance therapy for moderate-to-severe psoriasis: a phase III, multicentre, double-blind trial. Lancet 2005; 366: 1367 - 1374

7 Newland MR, Weinstein A, Kerdel F. Rapid response to infliximab in severe pustular psoriasis, von Zumbusch type. Int J Dermatol 2002; 41: $449-452$

8 Schmick K, Grabbe J. Recalcitrant, generalized pustular psoriasis: rapid and lasting therapeutic response to antitumour necrosis factor-alpha antibody (infliximab). Br J Dermatol 2004; 150: 367

9 Routhouska SB, Sheth PB, Korman NJ. Long-term management of generalized pustular psoriasis with infliximab: case series. J Cutan Med Surg 2008; $12: 184-188$

10 Wolfe F, Michaud K. The effect of methotrexate and anti-tumor necrosis factor therapy on the risk of lymphoma in rheumatoid arthritis in 19562 patients during 89710 person-years of observation. Arthritis Rheum 2007; 56: 1433

11 Askling J, Fored CM, Baecklund E et al. Haematopoietic malignancies in rheumatoid arthritis: lymphoma risk and characteristics after exposure to tumour necrosis factor antagonists. Ann Rheum Dis 2005; 64: $1414-1420$

12 Gelfand JM, Berlin J, Van Voorhees A et al. The Risk of Lymphoma in Patients with Psoriasis. Journal of Investigative Dermatology 2006; 126 : 2194-2201

13 Brown ER, Charles KA, Hoare SA et al. A clinical study assessing the tolerability and biological effects of infliximab, a TNF-alpha inhibitor, in patients with advanced cancer. Ann Oncol 2008; 19: 1340-1346

14 Brown SL, Greene MH, Gershon SK et al. Tumor necrosis factor antagonist therapy and lymphoma development: twenty-six cases reported to the Food and Drug Administration. Arthritis Rheum 2002; Dec 46: $3151-3158$

15 Paul CF, Ho VC, McGeown C et al. Risk of Malignancies in Psoriasis Patients Treated with Cyclsporine: a 5 y Cohort Study. J Invest Dermatol 2003; 120: $211-216$

16 Väkevä L, Reitamo S, Pukkala E et al. Long-term follow-up of cancer risk in patients treated with short-term cyclosporine. Acta Derm Venereol 2008; 88: $117-120$ 